\title{
Elevations in EEG Power Spectra During a Go/No-go Task Following Acute Psychosocial Stress in Preschool Children
}

\author{
Ryan J. Giuliano, Chantal P. Delaquis, \& Leslie E. Roos \\ Department of Psychology, University of Manitoba, Winnipeg, MB
}

\begin{abstract}
Despite substantial research emphasizing the role of chronic stress on children's brain function, remarkably little is known about the more immediate effects of acute stress on brain activity. Here, we analyzed changes in power spectra of the electroencephalogram (EEG) during a Go/No-go task collected both before and after a laboratory psychosocial stressor validated for preschool children.

Significant increases in EEG power were observed broadly across bandwidths and electrode sites from pre- to post- manipulation measurements. Follow-up comparisons illustrate that pre- to post- increases in EEG power were more pronounced for the Stressor group than the Control group, particularly for the delta $(1-3 \mathrm{~Hz})$, high alpha $(9-12 \mathrm{~Hz})$ and beta $(12-20 \mathrm{~Hz})$ bandwidths. While exploratory, these results are amongst the first pieces of evidence documenting the effects of acute psychosocial stress on brain activity in young children.
\end{abstract}

Keywords: electroencephalogram, EEG, EEG power spectra, acute stress, psychosocial stress, inhibitory control, preschool children.

Data availability: All data for this study can be accessed on the open science framework at https://osf.io/ajnkzl. Please direct further inquiries to ryan.giuliano@umanitoba.ca. 


\section{Introduction}

Substantial research documents the vulnerability of the developing brain to repetitive stress, with alterations in the hippocampus, amygdala, and prefrontal cortex (PFC) linked to chronic stressors and early adversity, such as poverty (Johnson et al., 2016) and maltreatment (Teicher et al., 2016). Over time, repeated stress system activation is understood to influence structural and functional brain development, which in turn impacts how the brain will interact with future environmental stressors (McEwen et al., 2015). However, to date, limited work has examined the impact of acute stress on children's brain function. Gaining more knowledge on in-the-moment changes is important to understand how dynamic fluctuations in brain activity may be more or less adaptive in different contexts. Ultimately, such an understanding holds promise for informing early family-based interventions to support management and recovery from acute stressors before the accumulation of these stressors leads to the chronic, toxic variety of stress that is detrimental to long term well-being (Shonkoff et al., 2012).

The brain is a central component in the stress response system responsible for detecting environmental stressors and regulating physiological and behavioural responses to stress (McEwen \& Gianaros, 2010). Responding to stress involves bidirectional communication between the brain and the cardiovascular, immune and metabolic systems via the autonomic nervous system (ANS) and the endocrine system (McEwen \& Gianaros, 2010). Within the brain, the hippocampus, hypothalamus, amygdala, and the PFC are all key regions implicated in the stress response system. The hippocampus, located in the medial temporal lobe, supports memory and mood, is a target of glucocorticoids, and plays a role in the cessation of the stress response (McEwen, 2007). The hypothalamus is a key component in the hypothalamic-pituitary (HPA) axis, regulating the release of a cascade of hormones that prompt the release of cortisol (Reilly \& Gunnar, 2019). The amygdala has been implicating in responding to threats, and the PFC is involved in decision making, emotion regulation, and autonomic nervous system (ANS) function (McEwen \& Gianaros, 2010). The extent to which individuals differ in the engagement of the ANS in response to stress may influence susceptibility to the cognitive and neurological effects of acute stress (Roos et al., 2019).

In adults, acute stress has been shown to impair attention (Olver et al., 2015; Scholz et al., 2009; Vinski \& Watter, 2013) and inhibitory control processes (Jiang \& Rau, 2017; Roos et al., 2017b). However, conflicting evidence also suggests that, under some conditions, acute stress may enhance inhibitory control processes (Chang et al., 2020; Dierolf et al., 2017, 2018; Schwabe \& Wolf, 2013). Overall, meta-analytic evidence indicates that acute stress appears to have detrimental effects on working memory and cognitive flexibility, with its effects on inhibition being more nuanced. Inconsistent results may be due, in part, to differences in methodologies across studies, such as the lack of consistent benchmark criteria to indicate the presence of a stress response (i.e., cortisol reactivity; Jiang \& Rau, 2017; Roos et al., 2017c; Shields et al., 2016). Studies examining event-related potentials (ERPs) in healthy adults have found mixed results regarding whether acute stress facilitates or inhibits response inhibition. Work by Dierolf and colleagues $(2017 ; 2018)$ provides evidence that acute stress reliably enhances the magnitude of neural responses during response inhibition (i.e., N2 and P3), with mixed evidence of acute stress impacting behavioural measures of response inhibition (Dierolf et al., 2017, 2018). Other ERP work in adults has reported an opposite pattern of ERP results, with acute stress diminishing amplitudes of the N2 and P3 ERP components during response inhibition (Jiang \& Rau, 2017). However, comparable research has not been conducted in developmental samples of young children. A recently validated laboratory stressor paradigm shows promising results in stimulating an acute stress response in preschool-aged children (Roos et al., 2017a), thus allowing for further investigation of the effects of acute stress on neural functioning.

Given the lack of research to date on the effects of acute stress on neural function in children, we were interested in whether changes in electroencephalogram (EEG) power spectra during a childadapted Go/No Go task (GNG) would occur before and after a psychosocial stressor. EEG serves as a 
particularly useful tool for investigating the effects of acute stress on neural functioning, as it is relatively non-invasive and more feasible with young children than magnetic resonance imaging (Chandra et al., 2017). EEG power spectra in children are typically examined through canonical frequency bands consisting of delta (1-4 Hz), theta (4-6 Hz), low alpha (6-9Hz), high alpha $(9-12 \mathrm{~Hz})$, and beta $(12-20$ $\mathrm{Hz}$ ), with alpha estimated separately for lower and higher frequency ranges to account for pronounced

developmental changes in alpha power (Marshall et al., 2002). Further, the cognitive correlates of EEG bandwidths have been fairly well characterized, with delta power associated with general alertness (e.g., Sachdev et al., 2015), theta power related to selective attention and cognitive control (e.g., Buzzell et al., 2020; Orekhova et al., 2006), alpha power related to focused attention (e.g., Aftanas \& Golocheikine, 2001) and beta power related to active processing when on-task (e.g., Marek et al., 2018). Thus, changes in power spectra of EEG bandwidths as a function of acute stress might be informative as to the nature of systemic changes in cognition under stress.

Here, we examined whether a psychosocial stressor paradigm would lead to changes in EEG power spectra measured pre- and post-stressor in 4- to 6-year old children, as compared to a control group that completed an arousal-matched version of the task in the absence of the psychosocial stress component. A previous report from this sample noted that the stressor elicited activation of the HPA axis and sympathetic nervous system, along with deactivation of the parasympathetic nervous system, while no modulations of HPA or autonomic function were observed in the control group (Roos et al., 2017a). Given the lack of previous research on the effects of acute psychosocial stress on EEG power in children, we explored pre- to post-stress changes in EEG power across each of the predefined bandwidths of EEG. We predicted that acute psychosocial stress would be associated with robust differences in EEG power spectra at the post- relative to the pre-measurement, and that pre- to postdifferences in EEG power would be absent or substantially reduced in a group of children experiencing a control, non-stressful version of the induction task.

\section{Participants}

\section{Methods}

Eighty-four mother-child dyads volunteered to participate through community recruitment in a city in the Pacific Northwest region of the United States. Children (age, $M=5.38$ years, $S D=.65$, range $=4.20$ to 6.71 years) were randomly assigned to a Control $(n=26,14$ female) or Stressor condition ( $n$ $=58,33$ female), with more children in the Stressor condition to permit examination of within-condition individual differences (see Roos et al., 2019). Pre-enrollment screening excluded children with a history of psychiatric disorders, developmental delays, or serious health problems. Mothers reported a range of household incomes (median $=\$ 25,000$ - $\$ 29,999$; range $<\$ 4,999-\$ 100,000+$ ) and education (median = some college or associate degree; range: less than high school, to graduate or professional degree). The final sample with usable EEG data pre- and post-stressor consisted of 64 participants $(n=42$, Stressor). As shown in Table 1 (below), there were no significant differences in sociodemographic variables (i.e., age, sex, household income, maternal education) between Stressor and Control groups (all $p s>.21$ ). In terms of maternal education, children from both groups were most likely to have mothers with some college experience or an associate degree (Stressor $=45.2 \%, n=19$; Control = $54.5 \%, n=12)$.

Table 1. Demographic characteristics of the Stressor and Control groups.

\begin{tabular}{lccc}
\hline & Stressor Group & Control Group & $p$ \\
\hline$n$ & 42 & 22 & \\
Sex & 24 female, 18 male & 12 female, 10 male & .845 \\
Age mean $(S D)$ & 5.35 years $(0.65)$ & $5.45(0.72)$ & .545 \\
Household Income & $\$ 25,000-\$ 29,999$ & $\$ 20,000-\$ 24,999$ & .217 \\
Maternal Education $(S D)$ & $3.34(1.15)$ & $3.05(1.02)$ & .329 \\
\hline
\end{tabular}

Note: Significance tests reflect results of independent samples $t$-tests with 62 degrees of freedom.

Maternal education values reflect responses on a scale of 1 to 5 , where $1=$ less than high school, $2=$ high school equivalent, $3=$ some college or associates degree, $4=$ college degree, $5=$ graduate or professional degree. 


\section{Measures}

Go/No-go task. Children performed a child-friendly version of a zoo-themed Go/No-go task (Grabell et al., 2017; Grammer et al., 2014), adapted to include negative feedback on Go trails and positive or negative feedback on No-go trials (see Roos et al., 2019). First, children were told a short story about animals escaping a zoo. Next, children were told that their job was to press a button as quickly as possible to catch the animals, each time an animal picture (Go stimuli) was presented on the screen. However, if a chimpanzee picture was presented (No-go stimuli), they were instructed to withhold a button press because the chimpanzees were helping to catch the other animals. Research assistants emphasized the importance of going quickly and praised the children for catching animals in order to increase the prepotency of the Go response. Children completed three practice blocks to ensure that they understood task instructions: 1) self-paced Go-only trials, 2) speeded response with Go-only trials, and 3) speeded response with mixed Go and No-go trials.

The task included two blocks of 60 trials each, with No-go stimuli presented $25 \%$ of the time. Children completed two task blocks pre- and post- stressor. During each trial, animal stimuli were presented for $1200 \mathrm{~ms}$, followed by a fixation cross for $1500 \mathrm{~ms}$ or until a response was made. The intertrial interval consisted of presentation of a fixation cross for $500 \mathrm{~ms}$. On Go trials, when children responded correctly, they proceeded to the next trial; however, if they failed to respond quickly enough, an unhappy face was presented to indicate trial failure. Children were told that these unhappy faces indicated a need to respond faster on Go trials. Happy faces were presented for each correct No-go trial, and unhappy faces were presented for each incorrect trial.

Animal stimuli were adapted from previous Go/No-go task versions in order to have two distinct sets of animal stimuli (forest- and savanna-themed) for use pre- and post-stressor. The forest and savanna picture sets were presented in a counter-balanced order across subjects. Animal stimuli were all vertically cropped images emphasizing the animal's face, in an attempt to maximize ease of identifying the animal and consistency of stimuli, as well as to minimize horizontal eye movement. The forest set consisted of pictures of bears, deer, foxes, raccoons, wolves, and cougars, while the savanna set consisted of pictures of giraffes, lions, meerkats, zebras, and dingos.

Acute Stressor Manipulation. The stressor employed in the present study was a matching task adapted from previous research (Kryski et al., 2011; Tolep \& Dougherty, 2014) and described in detail elsewhere (Roos et al., 2017a). Briefly, across Stressor and Control conditions, children played a "matching game" in which they were asked to use a color-coded legend to match colored stickers to transportation types on a worksheet with 30 squares to complete. Children performed this task on three consecutive two-minute trials, but were not able to complete the task, because two minutes is an insufficient time for most 4 - 6-year-old children to complete the worksheet. Two children were close to finishing the worksheet within two minutes, so the task was covertly stopped $\sim 10$ seconds short and the stop light flashed, so that these participants experienced task failure.

In the Stressor condition, children picked a desired prize to win for successfully completing a worksheet. The assessor, an unfamiliar adult who used stern, flat affect, operated a stoplight that was set to green for 90 seconds, then yellow for 30 seconds, and immediately afterwards to red, accompanied with a loud beep signaling the end of a trial. The assessor provided negative feedback twice each trial (e.g., "You need to go faster.") and after three trial failures, children were told they did not earn their prize. Next, the assessor left the room and a friendly, familiar assessor returned. Following the end of the experiment (i.e., after the post-stress GNG), the child was debriefed about the nature of the task and was told that they did an excellent job and earned their desired prize.

In the Control condition, facilitated by a friendly, familiar assessor, the children were told that after two minutes it was time to work on the next worksheet. There was no mention of prizes, winning, or losing. Notably, our previous work (Roos et al., 2017a) demonstrated that the matching task elicited significant between group differences in cortisol reactivity, with the Stressor group showing significant HPA activation that was absent in the Control group. Similarly, the Stressor group showed greater cardiac reactivity, seen as deactivation of the parasympathetic nervous system and activation of the sympathetic nervous system, relative to the Control group. 
Electroencephalogram (EEG). EEG was recorded using a 64-channel HydroCel Geodesic sensor net (Electrical Geodesics Inc., Eugene, OR), acquired via NetStation software at $500 \mathrm{~Hz}$. After recording, continuous EEG data was exported from NetStation in SimpleBinary format, then imported into the Matlab EEGLAB toolbox for processing. Continuous EEG files were then filtered using pop_eegfiltnew(), first high pass filtered at $0.1 \mathrm{~Hz}$ and then low pass filtered at $40 \mathrm{~Hz}$. After filtering, EEG data was subject to the clean_rawdata() function, which performs bad channel removal, bad window removal, and uses the artifact subspace reconstruction method to detect and remove non-brain artifacts (e.g., eye blinks, muscle activity, electrode movement). Each bad channel was replaced by a spherical interpolation of surrounding 'good' channels using the pop_interp() function. As the final step of preprocessing, EEG data was re-referenced to the average mastoids. Then, epochs of EEG were selected based on event codes demarcating the beginning and end of the pre- and post-manipulation GNG tasks. For each epoch, the spectopo() function was used to extract EEG power spectra pertaining to the delta $(1-4 \mathrm{~Hz})$, theta $(4-6 \mathrm{~Hz})$, low alpha $(6-9 \mathrm{~Hz})$, high $(9-12 \mathrm{~Hz})$, and beta $(12-20 \mathrm{~Hz})$ bandwidths. EEG power spectra values extracted from each electrode were natural log transformed using the $\log ()$ function before analysis. In order to reduce the number of comparisons being examined, electrodes were aggregated into anterior, central, and posterior composites. As shown in Figure 1 (below), anterior electrodes included four electrodes centered around electrode Fz, central electrodes included four electrodes centered around electrode $\mathrm{Cz}$ (but not including $\mathrm{Cz}$, as it was an online reference electrode), and posterior electrodes included four electrodes centered around electrode Pz.

Figure 1. Electrode montage depicting electrode aggregates calculated for Anterior, Central, and Posterior sites. Note that "REF Cz" was not included in the Central aggregate.

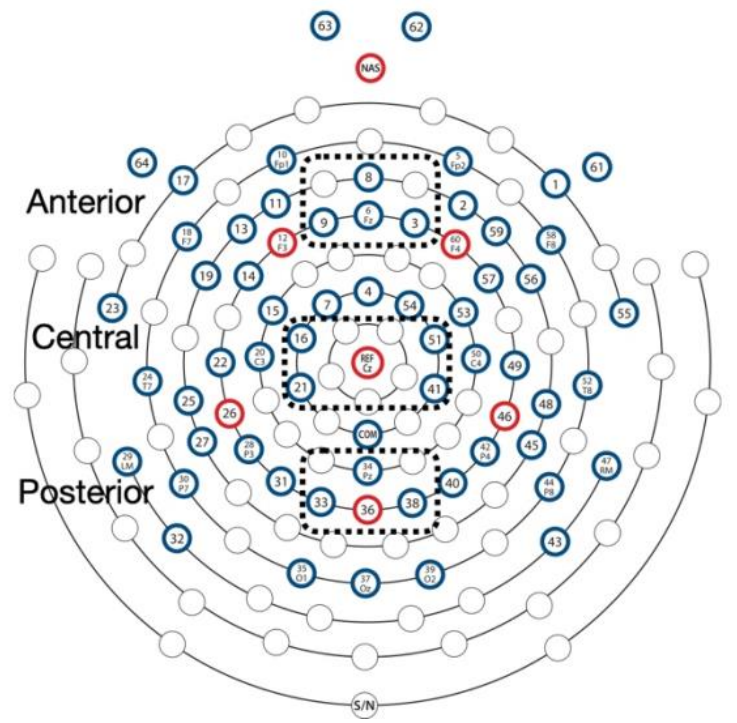

\section{Procedure}

Participants completed one 2-hour laboratory visit during which all measures were collected. Start times ranged from 9am to 3pm, with children awakening at least 1 hour prior and not eating 1 hour prior to the visit. Mother and child dyads arrived at the lab and were greeted by two assessors who escorted them to a confidential laboratory testing area. One assessor focused on engaging the child with their role as a 'Zookeeper Explorer' during the lab visit and animal related activities, including a coloring book and sticker chart describing the activities to be completed during the visit. Following a description of lab activities, written consent was obtained from the mother and verbal assent from the child. Next, the mother assisted the assessors in encouraging child comfort with the application of the EEG net along with electrodes for monitoring of cardiac physiology (see Roos et al., 2017a for full details on collection of cardiac and endocrine measures).

Testing began approximately 45 minutes after lab entry, with mother and child sitting in chairs across a table while facing each other but not making physical contact and watching a 5-minute video of neutral affect ocean scenes, for the assessment of baseline cardiac activity (Piferi et al., 2000). After the video, a research assistant escorted the mother out of the room to complete questionnaires, while 
another research assistant stayed in the room with the child participant to begin the "pre" Go/No-go task (2 task blocks). Afterwards, the child completed the matching task (either Stressor or Control condition), and then a 'post' Go/No-go task (2 task blocks). Following the post Go/No-go task, mother and child were reunited and additional laboratory activities were conducted (not described here). Notably, with the exception of the matching task, all laboratory procedures for the Stressor and Control condition were identical regarding assessments and children's interactions with their mothers and assessors.

\section{Analytic Plan}

All analyses were conducted in SPSS (Version 26.0). A repeated measures ANOVA was used to examine the effects of group (Stressor, Control) on EEG power at five different bandwidths (delta, theta, low alpha, high alpha, beta) by three electrode locations (frontal, central, posterior), across time (pre-manipulation, post-manipulation). Within the ANOVA, simple effects of time (pre-to-post) within group were used to examine changes in EEG power from pre- to post-manipulation for each bandwidth and electrode aggregate location separately for the two groups. Greenhouse-Geisser corrected degrees of freedom were used for all reported effects. Raincloud plots were generated using R ( $v$. 3.5.3; R core team, 2019) and the RainCloudPlots package (Allen et al., 2019), with data and code 1 to recreate the plot available on OSF (https://osf.io/ajnkz/).

\section{Descriptive Statistics}

\section{Results}

Zero-order correlations between sociodemographic variables and EEG power for pre- and postmanipulation measures, at each bandwidth and electrode location, are shown in the supplementary materials (Supplemental Table 1). Amongst sociodemographic variables, only household income and maternal education showed evidence of a significant association $[r(63)=.441, p=.001]$, such that higher household income tended to co-occur with higher maternal education. When examining associations with EEG power, sex was the only sociodemographic variable to demonstrate significant correlations. Sex was positively correlated with theta and low alpha power at the posterior electrode aggregate. For theta power, the correlation with sex was significant both pre-manipulation $[r(63)=.288, p=.021]$ and post-manipulation $[r(63)=.334, p=.007]$. For low alpha power, the correlation with sex was significant post-manipulation $[r(63)=.259, p=.039]$ and nonsignificant but trending in the same direction premanipulation $[r(63)=.244, p=.052]$.

\section{Effects of Acute Stress on EEG Power}

Results of the repeated measures ANOVA reveal significant main effects of time $[F(1,62)=20.21, p<$ $\left..001, \eta_{2 p}=.246\right]$, bandwidth $[F(4,248)=1737.55, p<$ $\left..001, \eta_{2 p}=.966\right]$, and electrode aggregate location $[F(2$, $124)=245.06, p<.001, \eta 2 p=.798]$ on EEG power spectra. Generally, EEG power spectra estimates were greater during the GNG task after the stressor/control manipulation $(M=1.56, S D=.34)$ relative to the $G N G$ task administered before the manipulation $(M=1.44, S D$ $=.38$ ). Comparing across bandwidths, EEG power decreased from lower to higher frequency bandwidths [delta $(M=2.79, S D=.34)$, theta $(M=2.24, S D=.40)$,

\begin{tabular}{|c|c|c|c|c|}
\hline & & Power & Power & \\
\hline Bandwidth & Location & Pre & Post & $p$ \\
\hline Delta & Anterior & 2.89 & 2.92 & .317 \\
\hline & Central & 2.25 & 2.34 & $.014^{\star}$ \\
\hline & Posterior & 3.14 & 3.17 & .302 \\
\hline Theta & Anterior & 2.19 & 2.30 & $<.001^{\star \star \star \star}$ \\
\hline & $\begin{array}{l}\text { Central } \\
\text { Posterior }\end{array}$ & $\begin{array}{l}1.74 \\
2.58\end{array}$ & $\begin{array}{l}1.91 \\
2.71\end{array}$ & $\begin{array}{l}<.001^{\star \star \star \star} \\
<.001^{\star \star \star \star}\end{array}$ \\
\hline Low Alpha & Anterior & 1.70 & 1.83 & $<.001^{\star * *}$ \\
\hline & $\begin{array}{l}\text { Central } \\
\text { Posterior }\end{array}$ & $\begin{array}{l}1.39 \\
2.21\end{array}$ & $\begin{array}{l}1.56 \\
2.38\end{array}$ & $\begin{array}{l}<.001^{\star \star \star *} \\
<.001^{\star \star \star *}\end{array}$ \\
\hline High Alpha & $\begin{array}{l}\text { Anterior } \\
\text { Central }\end{array}$ & $\begin{array}{l}0.66 \\
0.58\end{array}$ & $\begin{array}{l}0.75 \\
0.71\end{array}$ & $\begin{array}{l}.003^{\star \star *} \\
.001^{\text {k* }}\end{array}$ \\
\hline & Posterior & 1.32 & 1.42 & $.002^{\star \star}$ \\
\hline Beta & Anterior & -0.30 & -0.16 & $<.001^{\star \star \star \star}$ \\
\hline & $\begin{array}{l}\text { Central } \\
\text { Posterior }\end{array}$ & $\begin{array}{l}-0.64 \\
-0.05\end{array}$ & $\begin{array}{l}-0.51 \\
0.04\end{array}$ & $\begin{array}{l}.001^{\star \star \star} \\
.005^{\star \star \star}\end{array}$ \\
\hline
\end{tabular}

NOTE: all pairwise comparisons tested at $F(1,62)$ ${ }^{*}, p<.05 ;{ }^{* *}, p<.01 ; * * *, p<.001$ 
low alpha $(M=1.84, S D=.49)$, high alpha $(M=.91, S D=.43)$, beta $(M=-.27, S D=.34)$. Examining EEG power across as a function of location on the scalp, EEG power was lowest at the central electrode aggregate $(M=1.13, S D=.38)$ and greatest at the posterior aggregate $(M=1.89, S D=.46$; anterior, $M=1.48, S D=.29)$.

However, these main effects were qualified by higher-order interactions of time $\mathrm{x}$ bandwidth $\left[F(4,248)=9.83, p<.001, \eta_{2 p}=.137\right]$, bandwidth $\times$ electrode location $\left[F(8,496)=53.34, p<.001, \eta_{2 p}\right.$ $=.462]$, and time $x$ bandwidth $x$ electrode location $[F(8,496)=4.48, p<.001, \eta 2 p=.067]$. Collapsing across group, EEG power spectra increased from pre- to post-manipulation for all bandwidths and electrode aggregate locations except for delta power at anterior $(p=.317)$ and posterior electrodes $(p=$ .302). Full details of pre-post effects on EEG power are shown in Table 2. All omnibus main effects and interactions including the group factor were non-significant (all ps > .19).

\section{Exploratory Simple Effects of Time by Group}

Consistent with our objective to characterize effects of acute stress on EEG power, we followed up with a priori examination of pairwise comparisons of pre- vs. post-manipulation power spectra for each bandwidth and electrode location. Results are summarized by EEG bandwidth below and in Table 3. As shown in the supplemental materials (Supplemental Table 2), there were no significant differences in EEG power between groups at the pre-manipulation measure (all ps > .21), suggesting that any pre-post differences in EEG power between group were not due to pre-manipulation levels.

For the delta band $(1-4 \mathrm{~Hz})$, no differences in pre- vs. post- EEG power were observed in the Control group (all ps $>$.38). In contrast, the Stressor group showed significant increases in delta power from pre- to post-stressor at central $(p=$ $.003)$ and posterior electrodes $(p=.029)$.

For the theta band $(4-6 \mathrm{~Hz})$ and low alpha band (6-9 Hz), both groups showed robust increases in EEG power from pre- to post-manipulation at most electrode aggregates. Effects were nominally larger in the Stressor group (all ps <.001), with the Control group showing smaller pre-post mean differences (theta, $p s>.01$; alpha, ps $>$.009) and not showing significant changes in theta power at the posterior electrode cluster $(p=.069)$
Table 3. Pairwise Comparisons of Pre- vs. Post- Manipulation EEG Power Performed Separately for the Stressor and Control Groups.

\begin{tabular}{|c|c|c|c|c|c|c|c|}
\hline \multirow[b]{2}{*}{ Bandwidth } & \multirow[b]{2}{*}{ Location } & \multicolumn{3}{|c|}{$\begin{array}{l}\text { Stressor Group (n=42) } \\
\text { Power Power }\end{array}$} & \multicolumn{3}{|c|}{$\begin{array}{l}\text { Control Group }(\mathbf{n = 2 2}) \\
\text { Power Power }\end{array}$} \\
\hline & & Pre & Post & $p$ & Pre & Post & $p$ \\
\hline \multirow[t]{3}{*}{ Delta } & Anterior & 2.89 & 2.93 & .196 & 2.90 & 2.91 & .765 \\
\hline & Central & 2.21 & 2.35 & $.003^{\star \star \star}$ & 2.28 & 2.33 & .381 \\
\hline & Posterior & 3.12 & 3.20 & $.029^{\star}$ & 3.16 & 3.14 & .741 \\
\hline \multirow[t]{3}{*}{ Theta } & Anterior & 2.17 & 2.29 & $<.001^{\star \star \star \star}$ & 2.20 & 2.30 & $.014^{\star}$ \\
\hline & Central & 1.69 & 1.89 & $<.001^{\star \star \star}$ & 1.80 & 1.92 & $.026^{*}$ \\
\hline & Posterior & 2.55 & 2.72 & $<.001^{\star \star \star *}$ & 2.61 & 2.70 & .069 \\
\hline \multirow[t]{3}{*}{ Low Alpha } & Anterior & 1.67 & 1.80 & $<.001^{\star \star \star *}$ & 1.74 & 1.86 & $.009^{\star \star}$ \\
\hline & Central & 1.32 & 1.53 & $<.001^{\star \star *}$ & 1.45 & 1.59 & $.015^{\star}$ \\
\hline & Posterior & 2.18 & 2.39 & $<.001^{\star \star * *}$ & 2.24 & 2.36 & $.020^{*}$ \\
\hline \multirow[t]{3}{*}{ High Alpha } & Anterior & 0.66 & 0.73 & $.034^{\star}$ & 0.67 & 0.77 & $.025^{\star}$ \\
\hline & Central & 0.54 & 0.70 & $.001^{\star \star}$ & 0.61 & 0.71 & .108 \\
\hline & Posterior & 1.30 & 1.44 & $<.001^{\star \star \star *}$ & 1.33 & 1.40 & .229 \\
\hline \multirow[t]{3}{*}{ Beta } & Anterior & -0.25 & -0.12 & $.001^{\star \star \star}$ & -0.35 & -0.20 & $.002^{\star \star}$ \\
\hline & Central & -0.66 & -0.47 & $<.001^{\star \star *}$ & -0.62 & -0.54 & .194 \\
\hline & Posterior & -0.05 & 0.07 & $.003^{\star \star}$ & -0.05 & -0.02 & .193 \\
\hline
\end{tabular}

For the high alpha band $(9-12 \mathrm{~Hz})$ and beta band $(12-20 \mathrm{~Hz})$, differences in pre- vs. post- EEG power for the Control group were only observed at anterior electrodes (high alpha, $p=.025$; beta, $p=$ .002). In contrast, the Stressor group showed significant increases in high alpha power and beta power from pre- to post-stressor at anterior (high alpha, $p=.034$; beta, $p=.001$ ), central (high alpha, $p=.001$; beta, $p<.001$ ), and posterior electrodes (high alpha, $p<.001$; beta, $p=.003$ ).

To illustrate the differences in pre-post changes in EEG power between groups, raincloud plots of post- minus pre- manipulation EEG power are shown below for each group (Figure 2). Across most bandwidths and scalp locations, the distribution of the Stressor group shows relatively greater frequency of positive values. 


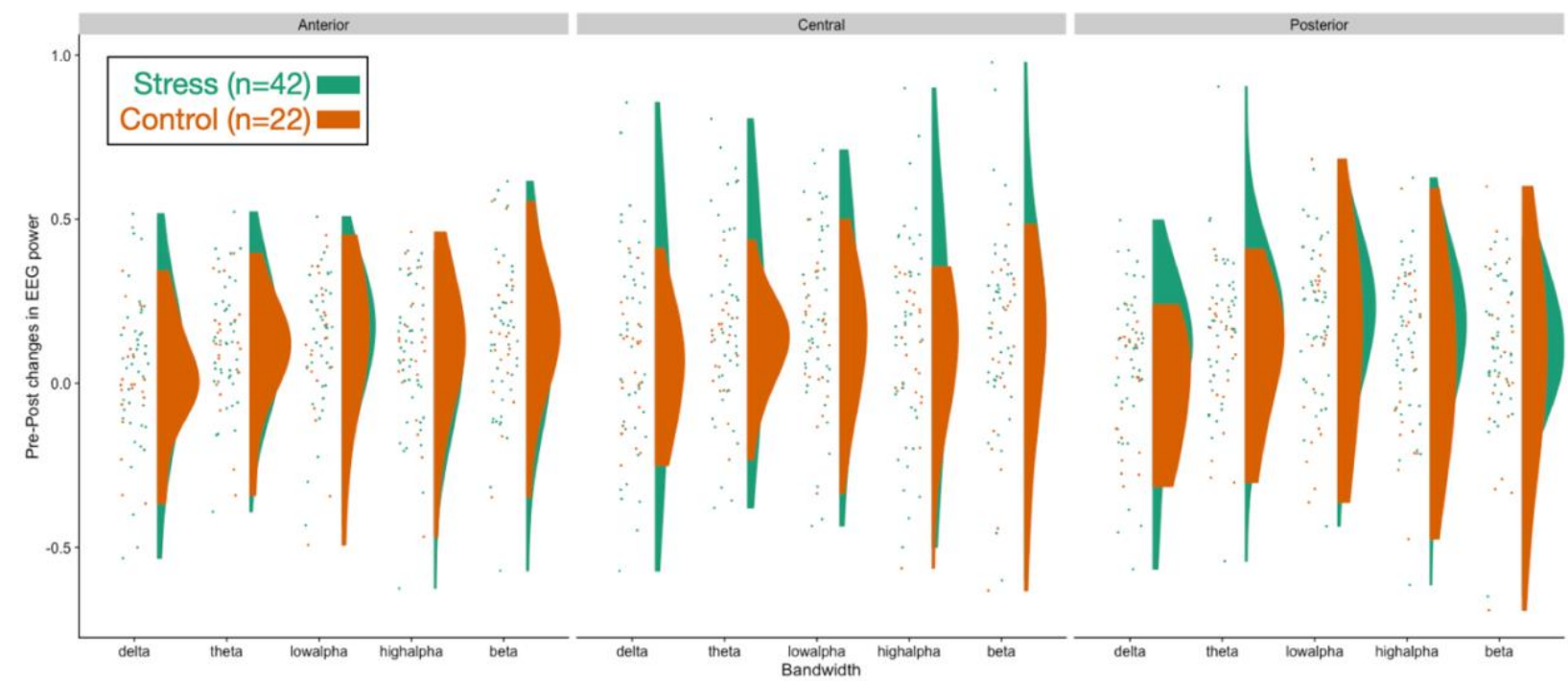

Figure 2. Raincloud plots of pre-post changes in EEG power for the Stressor and Control groups at the Anterior, Central, and Posterior electrode aggregates. Data points represent difference scores of EEG power (post minus pre).

\section{Discussion}

Here, we examined changes in EEG power in a sample of preschool children during a Go/No-go task performed before and after an acute stressor or a control task. Omnibus tests of group by time interactions on EEG power were not significant; however, a priori simple effects of EEG power changes from pre- to post- manipulation were examined for each group. Whereas the Stressor group showed robust pre-post changes in EEG power across bandwidths and across anterior to posterior electrode aggregates, EEG power changes in the Control group were primarily seen for the theta and low alpha bandwidths and most consistent at the anterior scalp measures. While these results should be interpreted with caution due to the relatively small sample size and exploratory nature of the analyses herein, these results are consistent with other studies of adults which report modulations of EEG power as a function of acute stress. Below, we discuss potential implications of these findings and offer suggestions for future studies of acute stress and EEG activity, particularly in younger populations.

Consistent with previous research on adults (Putman et al., 2014), children in the Stressor group showed post-stressor increases in theta and alpha power across electrode sites. While the Control group also showed significant increases in theta and alpha power, we observed some evidence that these effects were less robust than the Stressor group. Whereas the Stressor group showed significant increases in EEG power at theta (4-6 Hz), low alpha (6-9 Hz), and high alpha (9-12 Hz) bandwidths at all electrode locations, the Control group did not show significant increases in theta or high alpha power at posterior electrodes, nor for high alpha power at central electrodes. The observation of significant changes in EEG power in the Control group is not surprising, considering that children were performing the second block of a GNG task (with the first performed pre-manipulation), and given evidence from previous studies documenting repetition effects on brain activity when cognitive tasks are repeated (e.g., Ceballos et al., 2012). Based on the findings presented here, the unique effects of acute stress on brain activity might be most pronounced at central to posterior regions of cortex. However, any assumptions about underlying neural sources from EEG at the scalp should be taken with much caution.

A primary difference in EEG power changes between groups was the observation of pre-post increases in delta power exclusively in the Stressor group. This finding is consistent with previous studies of adults documenting associations between life stress and arousal with increases in delta 
power (Hasenfratz \& Bättig, 1992; A. C. Marshall \& Cooper, 2017) suggesting that increases in delta power for the Stressor group may reflect increases in psychosocial stress and arousal not experienced by children undergoing the control version of the matching task. In the supplemental materials, we present correlations between changes in EEG power and measures of physiological stress and arousal. We found weak to non-existent effects relating changes in EEG power in any bandwidth or electrode site with reactivity measures of cortisol, heart rate variability, or pre-ejection period for the Stressor group2 (see Supplemental Table 3); however, we note that our study is underpowered to detect such associations, highlighting the need for future research in this domain.

Another primary difference in results between groups was the observation of increases in posterior EEG power across all bandwidths in the Stressor group, while the Control group only showed a significant increase in 1 of 5 bandwidths (low alpha). This result may reflect an analogous mechanism to two other studies finding EEG power differences at posterior scalp locations in samples of young children who have experienced different forms of chronic stress (Blaisdell, Barker, Giuliano, \& Fisher, in revision; Curtis \& Cicchetti, 2007). A comparison of foster children and a community control sample found that differences in asymmetry of resting low alpha power $(6-9 \mathrm{~Hz})$ were only significant at posterior electrode sites (Blaisdell et al.). A separate study comparing school-age children who had experienced maltreatment versus nonmaltreated children found that resting EEG power at posterior electrodes in a broad alpha bandwidth $(7-11 \mathrm{~Hz}$ for $6-8$ year olds; 8-13 Hz for 9-12 year olds) differentiated between the two groups (Curtis \& Cicchetti, 2007). Since neither study examined EEG bandwidths other than alpha, it remains unclear whether analogous modulations of theta and beta power as a function of psychosocial stress would be observed in those samples.

Similar to the delta power results reported above, pre-post increases in beta power were somewhat exclusive to the Stressor group. The Control group showed increases in beta power only at the anterior electrode aggregate (central, $p=.194$; posterior, $p=.193$ ), while the Stressor group showed robust increases at the anterior $(p=.001)$, central $(p<.001)$, and posterior $(p=.003)$ aggregates. To our knowledge, there is limited work examining effects of acute stress on beta power, although some studies of adults report modulations of beta power during tasks imposing a high cognitive load (e.g., Putman et al., 2014). It should be noted that much of this research has focused on frontal beta power, leaving beta power at central and posterior locations of the scalp less understood in the context of acute stressors.

A final result of note is the observation of correlations between sex and EEG power at posterior electrode aggregates. Averaging across group, we observed some evidence that both theta and low alpha power may be more pronounced in female than male preschoolers. These correlations should be interpreted with caution given the large number of correlations examined and the lack of an a priori prediction for such an effect; yet, the consistency of directionality in the association between sex and EEG power in the theta and low alpha bandwidths is compelling and worth exploring in future studies. Previous research with an adult sample has similarly documented greater on-task theta power in females relative to males, however no differences were observed in the alpha band (Kober et al., 2016). In contrast, a study of sex differences in preadolescent children found weak to no evidence of sex differences in EEG power (Martinović et al., 1998).

There are several limitations to this work. Due to the novel nature of acute stress EEG research in children, this was a highly exploratory study without specific predictions about how EEG bandwidths would be modulated from pre to post stressor. Our lack of predictions was primarily due to the confluence of a dearth of existing research on either 1) the effects of acute psychosocial stress on EEG activity or 2) EEG power in children across bandwidths during cognitive tasks. To the latter point, much research on EEG power in children has focused on resting measures, when children are asked to sit still and alternate between 30 -second periods of having their eyes open or closed. Similarly, research

2 Cortisol reactivity was associated with pre-post changes in low alpha power at the anterior electrode aggregate; yet, this was only one of 45 correlations that reach significance at $p<.05$. 
on EEG power has traditionally focused on asymmetries in power between electrodes over the left and right hemispheres of the brain, with a particular emphasis on alpha power (Coan \& Allen, 2004). An expansion of EEG research to domains beyond asymmetries in power spectra is crucial, given the relatively poor understanding of the functional correlates of canonical EEG bandwidths to date. We hope this work is a step towards a data-driven approach of characterizing factors that modulate EEG power across bandwidths.

Given that our results are from on-task measures, it is possible that the EEG power changes observed here are confounded with modulations of oscillatory activity, particularly those in the theta and alpha bandwidth, that may be driven by repeated and timely presentation of visual stimuli in the GNG task. However, we view this as a strength of our experimental design, as we are most interested in characterizing how acute stress impacts brain and behaviour during active cognition, and since we utilized a between-subjects design wherein all participants experienced the exact same laboratory visit other than the procedural details of the matching task between GNG blocks. It is possible that acute stress may selectively modulate how children attend to the visual stimuli, leading to EEG power changes driven by oscillatory entrainment. Yet, previous research with healthy adults has demonstrated effects of acute stress on brain activity measured via event-related potentials during a GNG task similar to the task employed here (Dierolf et al., 2017, 2018). The results reported by Dierolf and colleagues $(2017 ; 2018)$ have parallel implications to our observation of delta power increases selective to the Stressor group, in suggesting that acute stress leads to arousal-related modulations of brain activity.

There are also methodological details of our EEG analyses that should be considered when interpreting the present results. From the sample of 82 children who completed the pre- and postmanipulation GNG task, only 64 children had usable EEG data, with 14 participants lost from the Stressor group and 4 participants lost from the Control group. We did not have the foresight to record video of the participants during the GNG task, but anecdotal reports from our research team suggest that children in the Stressor group were much less likely to sit still during the post-stressor GNG task when EEG was recorded. Thus, there is likely self-selection in the Stressor group, such that participants with usable EEG data were those children able to sit still enough for a usable EEG recording. This raises the empirical question whether the final sample in either group is biased towards children with better self-regulation abilities, which is an important source of individual differences in inhibitory control performance measured during GNG tasks. We note that this is likely a confound in most neuroimaging studies of children, which involve relatively rigid control of body and eye movements and call for more systematic examination into such potential self-selection biases.

Finally, we would like to call attention to the fact that our EEG data were processed using the cleanrawdata() function within EEGLAB. The cleanrawdata() function involves more preprocessing of raw data than has been advocated for by many EEG methodologists, including an automated correction of EEG data to remove activity fitting the profile of a non-brain artifact, which may have introduced systematic bias into the results. While it is theoretically possible that acute stress systematically modulates factors that interact with such an automated processing methodology, it seems more likely that any systematic bias from the cleanrawdata() function would be present in both the Stressor and Control groups. Further, developmental scientists have increasingly argued for the utility of such automated methods for processing EEG in samples of young children prone to movement and other non-brain artifacts in their EEG data (e.g., Debnath et al., 2020; Gabard-Durnam et al., 2018).

In summary, these results present preliminary evidence that psychosocial stress modulates the EEG power of preschoolers during cognitive performance. The changes in EEG power observed were broad both in terms of the bandwidths affected and regions of the scalp demonstrating significant power changes. Relative to a control group, unique effects of stress appear to be most pronounced for the delta band and across bandwidths at central and posterior regions of the scalp. These findings add to a small body of research documenting effects of acute stress on brain function. More research is needed to add specificity to neural mechanisms that may be impacted by acute stress and to identify how such impacts may vary across age, sex, and other sociodemographic variables. 


\section{References}

Allen, M., Poggiali, D., Whitaker, K., Marshall, T. R., \& Kievit, R. A. (2019). Raincloud plots: a multiplatform tool for robust data visualization. Wellcome open research, 4.

https://doi.org/10.12688/wellcomeopenres.15191.1

Aftanas, L. I., \& Golocheikine, S. A. (2001). Human anterior and frontal midline theta and lower alpha reflect emotionally positive state and internalized attention: High-resolution EEG investigation of meditation. Neuroscience Letters, 310(1), 57-60. https://doi.org/10.1016/S0304-3940(01)02094-8

Blaisdell, K. N., Barker, T. V., Giuliano, R. J., \& Fisher, P. A. (in revision). Alpha electroencephalogram (EEG) asymmetry among maltreated toddlers in foster care.

Buzzell, G. A., Troller-Renfree, S. V., Wade, M., Debnath, R., Morales, S., Bowers, M. E., Zeanah, C. H., Nelson, C. A., \& Fox, N. A. (2020). Adolescent cognitive control and mediofrontal theta oscillations are disrupted by neglect: Associations with transdiagnostic risk for psychopathology in a randomized controlled trial. Developmental Cognitive Neuroscience, 43, 100777. https://doi.org/10.1016/j.dcn.2020.100777

Ceballos, N. A., Giuliano, R. J., Wicha, N. Y. Y., \& Graham, R. (2012). Acute stress and event-related potential correlates of attention to alcohol images in Social drinkers. Journal of Studies on Alcohol and Drugs, 73(5), 761-771. https://doi.org/10.15288/jsad.2012.73.761

Chandra, S., Jaiswal, A., Singh, R., Jha, D., \& Mittal, A. (2017). Mental stress: Neurophysiology and its regulation by Sudarshan Kriya Yoga. International Journal of Yoga, 10(2), 67. https://doi.org/10.4103/0973-6131.205508

Chang, J., Hu, J., Li, C.-S. R., \& Yu, R. (2020). Neural correlates of enhanced response inhibition in the aftermath of stress. Neurolmage, 204, 116212. https://doi.org/10.1016/j.neuroimage.2019.116212

Coan, J. A., \& Allen, J. J. (2004). Frontal EEG asymmetry as a moderator and mediator of emotion. Biological psychology, 67(1-2), 7-50. https://doi.org/10.1016/j.biopsycho.2004.03.002

Curtis, W. J., \& Cicchetti, D. (2007). Emotion and resilience: A multilevel investigation of hemispheric electroencephalogram asymmetry and emotion regulation in maltreated and nonmaltreated children. Development and Psychopathology, 19(3), 811-840. https://doi.org/10.1017/S0954579407000405

Debnath, R., Buzzell, G. A., Morales, S., Bowers, M. E., Leach, S. C., \& Fox, N. A. (2020). The Maryland analysis of developmental EEG (MADE) pipeline. Psychophysiology, e13580. https://doi.org/10.1111/psyp.13580

Dierolf, A. M., Fechtner, J., Böhnke, R., Wolf, O. T., \& Naumann, E. (2017). Influence of acute stress on response inhibition in healthy men: An ERP study. Psychophysiology, 54(5), 684-695. https://doi.org/10.1111/psyp.12826

Dierolf, A. M., Schoofs, D., Hessas, E.-M., Falkenstein, M., Otto, T., Paul, M., Suchan, B., \& Wolf, O. T. (2018). Good to be stressed? Improved response inhibition and error processing after acute stress in young and older men. 119, 434-447. https://doi.org/10.1016/j.neuropsychologia.2018.08.020

Gabard-Durnam, L. J., Mendez Leal, A. S., Wilkinson, C. L., \& Levin, A. R. (2018). The Harvard Automated Processing Pipeline for Electroencephalography (HAPPE): Standardized Processing Software for Developmental and High-Artifact Data. Frontiers in Neuroscience, 12, 97. https://doi.org/10.3389/fnins.2018.00097

Grabell, A. S., Olson, S. L., Tardif, T., Thompson, M. C., \& Gehring, W. J. (2017). Comparing SelfRegulation-Associated Event Related Potentials in Preschool Children with and without High Levels of Disruptive Behavior. Journal of Abnormal Child Psychology, 45(6), 1119-1132. https://doi.org/10.1007/s10802-016-0228-7

Grammer, J. K., Carrasco, M., Gehring, W. J., \& Morrison, F. J. (2014). Age-related changes in error processing in young children: A school-based investigation. https://doi.org/10.1016/j.dcn.2014.02.001

Hasenfratz, M., \& Bättig, K. (1992). Action profiles of smoking and caffeine: Stroop effect, EEG, and peripheral physiology. Pharmacology, Biochemistry and Behavior, 42(1), 155-161. https://doi.org/10.1016/0091-3057(92)90459-S

Jiang, C., \& Rau, P.-L. P. (2017). The detrimental effect of acute stress on response inhibition when 
exposed to acute stress. NeuroReport, 28(14), 922-928.

https://doi.org/10.1097/WNR.0000000000000859

Johnson, S. B., Riis, J. L., \& Noble, K. G. (2016). State of the art review: Poverty and the developing brain. Pediatrics, 137(4). https://doi.org/10.1542/peds.2015-3075

Kober, S. E., Reichert, J. L., Neuper, C., \& Wood, G. (2016). Interactive effects of age and gender on EEG power and coherence during a short-term memory task in middle-aged adults. Neurobiology of aging, 40, 127-137. https://doi.org/10.1016/j.neurobiolaging.2016.01.015

Kryski, K. R., Smith, H. J., Sheikh, H. I., Singh, S. M., \& Hayden, E. P. (2011). Assessing stress reactivity indexed via salivary cortisol in preschool-aged children. Psychoneuroendocrinology, 36(8), 1127-1136. https://doi.org/10.1016/j.psyneuen.2011.02.003

Marek, S., Tervo-Clemmens, B., Klein, N., Foran, W., Ghuman, A. S., \& Luna, B. (2018). Adolescent development of cortical oscillations: Power, phase, and support of cognitive maturation. PLOS Biology, 16(11), e2004188. https://doi.org/10.1371/journal.pbio.2004188

Marshall, A. C., \& Cooper, N. R. (2017). The association between high levels of cumulative life stress and aberrant resting state EEG dynamics in old age. Biological Psychology, 127, 64-73. https://doi.org/10.1016/j.biopsycho.2017.05.005

Marshall, P. J., Bar-Haim, Y., \& Fox, N. A. (2002). Development of the EEG from 5 months to 4 years of age. Clinical Neurophysiology, 113(8), 1199-1208. https://doi.org/10.1016/S1388-2457(02)001633

Martinović, Z., Jovanović, V., \& Ristanović, D. (1998). EEG power spectra of normal preadolescent twins. Gender differences of quantitative EEG maturation. Neurophysiologie Clinique/Clinical Neurophysiology, 28(3), 231-248. https://doi.org/10.1016/S0987-7053(98)80114-7

McEwen, B. S. (2007). Physiology and neurobiology of stress and adaptation: Central role of the brain. In Physiological Reviews (Vol. 87, Issue 3, pp. 873-904). https://doi.org/10.1152/physrev.00041.2006

McEwen, B. S., Bowles, N. P., Gray, J. D., Hill, M. N., Hunter, R. G., Karatsoreos, I. N., \& Nasca, C. (2015). Mechanisms of stress in the brain. Nature Neuroscience, 18(10), 1353-1363. https://doi.org/10.1038/nn.4086

McEwen, B. S., \& Gianaros, P. J. (2010). Central role of the brain in stress and adaptation: Links to socioeconomic status, health, and disease. In Annals of the New York Academy of Sciences (Vol. 1186, pp. 190-222). Blackwell Publishing Inc. https://doi.org/10.1111/j.1749-6632.2009.05331.x

Olver, J. S., Pinney, M., Maruff, P., \& Norman, T. R. (2015). Impairments of spatial working memory and attention following acute psychosocial stress. Stress and Health : Journal of the International Society for the Investigation of Stress, 31(2), 115-123. https://doi.org/10.1002/smi.2533

Orekhova, E. V., Stroganova, T. A., Posikera, I. N., \& Elam, M. (2006). EEG theta rhythm in infants and preschool children. Clinical Neurophysiology, 117(5), 1047-1062. https://doi.org/10.1016/j.clinph.2005.12.027

Piferi, R. L., Kline, K. A., Younger, J., \& Lawler, K. A. (2000). An alternative approach for achieving cardiovascular baseline: Viewing an aquatic video. International Journal of Psychophysiology, 37(2), 207-217. https://doi.org/10.1016/S0167-8760(00)00102-1

Putman, P., Verkuil, B., Arias-Garcia, E., Pantazi, I., \& Van Schie, C. (2014). EEG theta/beta ratio as a potential biomarker for attentional control and resilience against deleterious effects of stress on attention. Cognitive, Affective and Behavioral Neuroscience, 14(2), 782-791. https://doi.org/10.3758/s13415-013-0238-7

Reilly, E. B., \& Gunnar, M. R. (2019). Neglect, HPA axis reactivity, and development. In International Journal of Developmental Neuroscience (Vol. 78, pp. 100-108). Elsevier Ltd. https://doi.org/10.1016/j.ijdevneu.2019.07.010

Roos, L. E., Giuliano, R. J., Beauchamp, K. G., Berkman, E. T., Knight, E. L., \& Fisher, P. A. (2019). Acute stress impairs children's sustained attention with increased vulnerability for children of mothers reporting higher parenting stress. Developmental Psychobiology. https://doi.org/10.1002/dev.21915

Roos, L. E., Giuliano, R. J., Beauchamp, K. G., Gunnar, M., Amidon, B., \& Fisher, P. A. (2017a). Validation of autonomic and endocrine reactivity to a laboratory stressor in young children. Psychoneuroendocrinology, 77, 51-55. https://doi.org/10.1016/j.psyneuen.2016.11.023 
Roos, L. E., Knight, E. L., Beauchamp, K. G., Berkman, E. T., Faraday, K., Hyslop, K., \& Fisher, P. A. (2017b). Acute Stress Impairs Inhibitory Control based on Individual Differences in Parasympathetic Nervous System Activity. Biological Psychology, 125, 58. https://doi.org/10.1016/J.BIOPSYCHO.2017.03.004

Roos, L. E., Knight, E. L., Beauchamp, K. G., Giuliano, R. J., Fisher, P. A., \& Berkman, E. T. (2017c). Conceptual precision is key in acute stress research: A commentary on Shields, Sazma, \& Yonelinas, 2016. In Neuroscience and Biobehavioral Reviews (Vol. 83, pp. 140-144). Elsevier Ltd. https://doi.org/10.1016/j.neubiorev.2017.10.005

Sachdev, R. N. S., Gaspard, N., Gerrard, J. L., Hirsch, L. J., Spencer, D. D., \& Zaveri, H. P. (2015). Delta rhythm in wakefulness: Evidence from intracranial recordings in human beings. Journal of Neurophysiology, 114(2), 1248-1254. https://doi.org/10.1152/jn.00249.2015

Scholz, U., La Marca, R., Nater, U. M., Aberle, I., Ehlert, U., Hornung, R., Martin, M., \& Kliegel, M. (2009). Go no-go performance under psychosocial stress: Beneficial effects of implementation intentions. Neurobiology of Learning and Memory, 91(1), 89-92. https://doi.org/10.1016/j.nlm.2008.09.002

Schwabe, L., \& Wolf, O. T. (2013). Stress and multiple memory systems: from 'thinking' to 'doing.' Trends in Cognitive Sciences, 17(2), 60-68. https://doi.org/10.1016/J.TICS.2012.12.001

Shields, G. S., Sazma, M. A., \& Yonelinas, A. P. (2016). The effects of acute stress on core executive functions: A meta-analysis and comparison with cortisol. In Neuroscience and Biobehavioral Reviews (Vol. 68, pp. 651-668). Elsevier Ltd. https://doi.org/10.1016/j.neubiorev.2016.06.038

Shonkoff, J. P., Garner, A. S., Siegel, B. S., Dobbins, M. I., Earls, M. F., McGuinn, L., Pascoe, J., Wood, D. L., High, P. C., Donoghue, E., Fussell, J. J., Gleason, M. M., Jaudes, P. K., Jones, V. F., Rubin, D. M., Schulte, E. E., Macias, M. M., Bridgemohan, C., Fussell, J., ... Wegner, L. M. (2012). The lifelong effects of early childhood adversity and toxic stress. Pediatrics, 129(1). https://doi.org/10.1542/peds.2011-2663

Teicher, M. H., Samson, J. A., Anderson, C. M., \& Ohashi, K. (2016). The effects of childhood maltreatment on brain structure, function and connectivity. Nature Reviews Neuroscience, 17(10), 652. https://doi.org/ 10.1038/nrn.2016.111

Tolep, M. R., \& Dougherty, L. R. (2014). The conundrum of the laboratory: Challenges of assessing preschool-age children's salivary cortisol reactivity. Journal of Psychopathology and Behavioral Assessment, 36(3), 350-357. https://doi.org/10.1007/s10862-014-9410-9

Vinski, M. T., \& Watter, S. (2013). Being a grump only makes things worse: a transactional account of acute stress on mind wandering. Frontiers in Psychology, 4, 730.

https://doi.org/10.3389/fpsyg.2013.00730 


\section{Supplementary Materials}

Supplemental Table 1. Zero-order Correlations Between Sociodemographic Variables and EEG Power at Pre- and

Post- Manipulation and Each Bandwidth and Electrode Location.

\begin{tabular}{|c|c|c|c|c|}
\hline & Age & Sex & Income & Matern \\
\hline Age & - & .031 & -.054 & .009 \\
\hline Sex & - & - & .053 & .075 \\
\hline Income & - & - & - & $.441^{\star *}$ \\
\hline Maternal Education & - & - & - & - \\
\hline Pre- Delta Anterior & -.169 & .058 & -.110 & -.106 \\
\hline Pre- Delta Central & .019 & -.126 & -.169 & .041 \\
\hline Pre- Delta Posterior & .017 & $.234 t$ & -.207 & -.031 \\
\hline Pre- Theta Anterior & -.153 & .187 & -.149 & -.114 \\
\hline Pre- Theta Central & -.022 & .056 & -.194 & .028 \\
\hline Pre- Theta Posterior & .010 & $.288^{*}$ & -.215 & -.050 \\
\hline Pre- Low Alpha Anterior & $-.218 t$ & $.218 t$ & -.165 & -.186 \\
\hline Pre- Low Alpha Central & -.065 & .110 & -.163 & .037 \\
\hline Pre- Low Alpha Posterior & .001 & $.244 \mathrm{t}$ & $-.226 t$ & .013 \\
\hline Pre- High Alpha Anterior & -.053 & .214 & -.053 & -.069 \\
\hline Pre- High Alpha Central & .053 & .018 & -.072 & .107 \\
\hline Pre- High Alpha Posterior & .137 & $.220 \mathrm{t}$ & -.141 & .045 \\
\hline Pre- Beta Anterior & -.134 & -.041 & -.001 & -.143 \\
\hline Pre- Beta Central & -.024 & -.096 & -.063 & -.009 \\
\hline Pre- Beta Posterior & .080 & .183 & -.128 & -.035 \\
\hline Post- Delta Anterior & -.185 & -.050 & -.017 & -.133 \\
\hline Post- Delta Central & -.070 & -.114 & -.037 & -.030 \\
\hline Post- Delta Posterior & .006 & .203 & -.155 & -.065 \\
\hline Post- Theta Anterior & -.189 & .188 & -.075 & -.102 \\
\hline Post- Theta Central & -.076 & .094 & -.018 & .010 \\
\hline Post- Theta Posterior & .033 & $.334^{\star \star}$ & -.140 & .046 \\
\hline Post- Low Alpha Anterior & $-.215 t$ & $.211 \mathrm{t}$ & -.114 & -.154 \\
\hline Post- Low Alpha Central & -.075 & .120 & -.022 & .027 \\
\hline Post- Low Alpha Posterior & .052 & $.259^{\star}$ & -.144 & .046 \\
\hline Post- High Alpha Anterior & -.050 & $.231 \mathrm{t}$ & -.030 & -.098 \\
\hline Post- High Alpha Central & .079 & .014 & .067 & .049 \\
\hline Post- High Alpha Posterior & .180 & $.234 \mathrm{t}$ & -.072 & .050 \\
\hline Post- Beta Anterior & $-.210 \mathrm{t}$ & -.041 & .009 & -.170 \\
\hline Post- Beta Central & -.018 & -.049 & -.006 & -.052 \\
\hline Post- Beta Posterior & .132 & $.225 t$ & -.154 & -.052 \\
\hline
\end{tabular}

$\mathrm{t}, p<.1 ;{ }^{*}, p<.05 ;{ }^{* \star}, p<.01 ;{ }^{* \star \star}, p<.001$ 
Supplemental Table 2. Pairwise Comparisons of EEG Power by Group for Pre-Manipulation Levels at Each Bandwidth and Electrode Location.

\begin{tabular}{|c|c|c|c|c|}
\hline Bandwidth & Location & Stressor & Control & $p$ \\
\hline \multirow[t]{3}{*}{ Delta } & Anterior & 2.89 & 2.90 & .934 \\
\hline & Central & 2.21 & 2.28 & .507 \\
\hline & Posterior & 3.12 & 3.16 & .732 \\
\hline \multirow[t]{3}{*}{ Theta } & Anterior & 2.17 & 2.20 & .715 \\
\hline & Central & 1.69 & 1.80 & .337 \\
\hline & Posterior & 2.55 & 2.61 & .657 \\
\hline \multirow[t]{3}{*}{ Low Alpha } & Anterior & 1.67 & 1.74 & .521 \\
\hline & Central & 1.32 & 1.45 & .345 \\
\hline & Posterior & 2.18 & 2.24 & .708 \\
\hline \multirow[t]{3}{*}{ High Alpha } & Anterior & 0.66 & 0.67 & .921 \\
\hline & Central & 0.54 & 0.61 & .599 \\
\hline & Posterior & 1.30 & 1.33 & .831 \\
\hline \multirow[t]{3}{*}{ Beta } & Anterior & -0.25 & -0.35 & .213 \\
\hline & Central & -0.66 & -0.62 & .759 \\
\hline & Posterior & -0.05 & 0.05 & .969 \\
\hline
\end{tabular}

NOTE: all pairwise comparisons tested at $F(1,62)$. 
Supplemental Table 3. Zero-order Correlations Between Physiological Reactivity to the Matching Task and Changes in EEG Power for Each Bandwidth and

Electrode Location.

\begin{tabular}{lccccccc} 
& \multicolumn{3}{c}{ Stressor Group } & \multicolumn{3}{c}{ Control Group } \\
& Cortisol & HF-HRV & PEP & \multicolumn{3}{c}{ Cortisol } & HF-HRV \\
& .194 & -.056 & .092 & .237 & -.106 & -.105 \\
\hline Delta Anterior & .074 & -.084 & -.067 & .211 & -.196 & -.276 \\
Delta Central & -.038 & .126 & .220 & -.052 & -.336 & -.148 \\
Delta Posterior & .193 & -.092 & .008 & .281 & -.388 & -.125 \\
Theta Anterior & .162 & -.088 & -.148 & $.428 \mathrm{t}$ & $-.418 \mathrm{t}$ & -.204 \\
Theta Central & .240 & .053 & .165 & .260 & $-.423 \mathrm{t}$ & -.187 \\
Theta Posterior & $.326^{\star}$ & -.069 & -.068 & .327 & -.328 & -.133 \\
Low Alpha Anterior & .174 & -.152 & -.151 & $.478^{\star}$ & -.294 & -.194 \\
Low Alpha Central & .246 & -.140 & .009 & .331 & $-.419 \mathrm{t}$ & -.422 \\
Low Alpha Posterior & .219 & -.264 & -.045 & .335 & -.194 & -.143 \\
High Alpha Anterior & .076 & -.212 & -.117 & $.559^{\star}$ & -.112 & -.161 \\
High Alpha Central & $.294 \mathrm{t}$ & -.151 & .058 & .155 & -.193 & -.232 \\
High Alpha Posterior & .139 & -.088 & -.005 & .275 & -.116 & .120 \\
Beta Anterior & -.006 & -.039 & -.024 & .281 & -.193 & -.033 \\
Beta Central & .006 & -.063 & .041 & .171 & -.186 & -.091 \\
Beta Posterior & & & & & & \\
\end{tabular}

$\mathrm{t}, p<.1 ;{ }^{*}, p<.05 ; \mathrm{HF}-\mathrm{HRV}$ : high-frequency heart rate variability; PEP: pre-ejection period;

Participants with physiological reactivity values varied by group and measure:

Stressor group, cortisol = 40, HF-HRV = 36, PEP = 33; Control group, cortisol = 18, HF-HRV = 18, PEP = 12. 